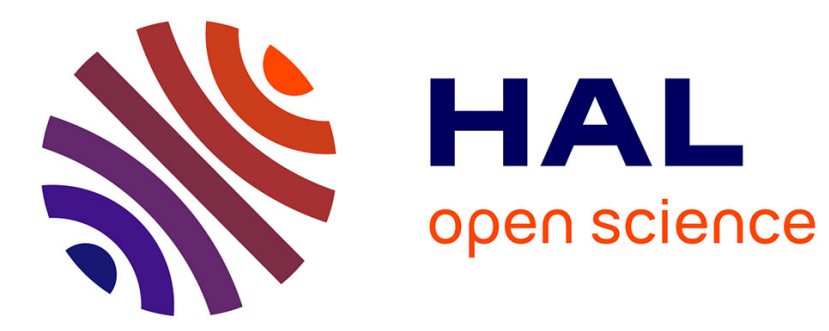

\title{
Scaling for a periodic forcing at the onset of intermittency
}

Françoise Argoul, A. Arnéodo

\section{To cite this version:}

Françoise Argoul, A. Arnéodo. Scaling for a periodic forcing at the onset of intermittency. Journal de Physique Lettres, 1985, 46 (19), pp.901-907. 10.1051/jphyslet:019850046019090100 . jpa-00232916

\section{HAL Id: jpa-00232916 https://hal.science/jpa-00232916}

Submitted on 1 Jan 1985

HAL is a multi-disciplinary open access archive for the deposit and dissemination of scientific research documents, whether they are published or not. The documents may come from teaching and research institutions in France or abroad, or from public or private research centers.
L'archive ouverte pluridisciplinaire HAL, est destinée au dépôt et à la diffusion de documents scientifiques de niveau recherche, publiés ou non, émanant des établissements d'enseignement et de recherche français ou étrangers, des laboratoires publics ou privés. 
Classification

Physics Abstracts

64.60F -05.90

\title{
Scaling for a periodic forcing at the onset of intermittency
}

\author{
F. Argoul and A. Arnéodo (*) \\ Centre de Recherche Paul Pascal, Université de Bordeaux I, Domaine Universitaire, \\ 33405 Talence Cedex, France
}

(Reçu le 28 juin 1985, accepté le 19 août 1985)

\begin{abstract}
Résumé. - Nous discutons de l'effet d'une excitation périodique sur un système qui transite vers le chaos par intermittence (de type I). Nous montrons que l'amplitude de l'excitation joue le rôle d'une variable d'échelle et que la longueur moyenne des phases laminaires présente des propriétés d'universalité au voisinage de la transition. A l'aide des techniques du Groupe de Renormalisation, nous calculons les exposants critiques correspondant aux différentes classes d'universalité et insistons sur le fait qu'ils ne dépendent pas de la fréquence d'entraînement. Nous présentons les résultats d'expérimentations numériques sur des systèmes discrets qui viennent corroborer ces prédictions théoriques.
\end{abstract}

\begin{abstract}
The effects of a periodic excitation on the transition to chaos via intermittency are considered. A renormalization group approach is used to predict the scaling behaviour of the average length of laminarity with respect to the amplitude of the forcing. The corresponding critical exponents are calculated for the different « universality classes " and found to be independent of the value of the external frequency. Numerical results on discrete systems are reported which corroborate these theoretical results.
\end{abstract}

The analogy between the transitions to chaos observed in dissipative dynamical systems [1-3] and critical phenomena [4] has been emphasized by many authors. The use of the renormalization group techniques [5] provides a unified and elegant description of the universal properties displayed by some of these scenarios and in particular by the cascade of period-doubling bifurcations [6-9] and intermittency [10-14]. In the presence of noise, one can carry on the analogy with phase transitions by considering the Lyapunov characteristic exponent as a « (dis)order » parameter which exhibits scaling behaviour close to the onset of chaos, and the external fluctuations as a disordering field on the deterministic dynamics [15-17]. Critical exponents were numerically computed for both period-doublings [18-21] and intermittency [12-14] and shown to be in remarkable agreement with the theoretical predictions obtained through a renormalization group analysis.

(*) Permanent address : Laboratoire de Physique théorique, Université de Nice, Parc Valrose, 06034 Nice Cedex, France. 
More recently, such an approach has been used to investigate the effects of a periodic forcing on the period-doubling cascade [22-24]. The main conclusion of [23, 24] is that the amplitude of the external excitation again behaves as a scaling variable. The critical exponent which in some sense characterizes the global instability [15-17] of the period-doubling cascade to such a perturbation is found to depend on the ratio of the driving to the external frequencies. Numerical experiments on discrete systems [23-25] not only corroborate these theoretical results but attest that there is a range in the driving frequency for which the period-doubling cascade is much more affected by a periodic forcing than by a random noise.

In this paper we propose to extend such a renormalization group formulation to the transition to chaos via intermittency in the presence of a periodic excitation. The inverse of the average length of the laminar episodes (quasiperiodic regime) increases from zero above the onset of chaos and displays some universal scaling properties with respect to the strength of the external forcing. We calculate the critical exponents corresponding to the different " universality classes » defined in the absence of forcing [12-14]. Our main result is that, on the contrary to perioddoublings, these critical exponents do not depend on the value of the external frequency. We report numerical investigations of 2-Dimensional mappings which bring strong experimental support to such a result and confirm that intermittency is much less sensitive to a periodic forcing than to external noise.

While the period-doubling cascade is based on the pitchfork bifurcation, intermittency results from a tangent (saddle-node) bifurcation. As originally discovered by Pomeau and Manneville [10], it consists of arbitrarily long episodes of nearly periodic behaviour separated by intermittent bursts. The simplest models to study such a transition to chaos are the following class of 1-D maps :

$$
f(x)=x+a|x|^{z}+\mu
$$

where $\mu$ accounts for a displacement from tangency $(\mu=0)$ and the exponent $z$ determines the « universality classes ». For small $\mu>0$, just beyond the onset of chaos, the duration of the laminar sequences $\left(x_{n} \approx 0\right)$ decreases like $\mu^{-(z-1) / z}$. Such a scaling behaviour comes out quite naturally from a renormalization group approach as developed in $[13,14]$. There exists an exact solution to the renormalization operation.

$$
g^{*}\left(g^{*}(x)\right)=\alpha^{-1} g^{*}(\alpha x)
$$

namely

$$
g^{*}(x)=x\left[1-(z-1) a \cdot \operatorname{sgn}(x)|x|^{z-1}\right]^{-1} /(z-1)
$$

with the rescaling factor :

$$
\alpha=2^{1 /(z-1)} .
$$

The stability of this fixed point is given by the spectrum of the linear part of the renormalization operator. The eigenfunction $h_{\lambda}(x)$ satisfies

$$
g^{*^{\prime}}\left(g^{*}(x)\right) \cdot h_{\lambda}(x)+h_{\lambda}\left(g^{*}(x)\right)=\lambda \cdot h_{\lambda}(\alpha x) / \alpha .
$$

The relevant eigenfunction corresponds to a shift from tangency, and is associated with the eigenvalue :

$$
\lambda_{1}=2^{z /(z-1)}
$$

which lies out of the unit circle. Then the critical exponents can be easily deduced from $\lambda_{1}$. In the vicinity of the chaotic threshold, the envelop of the Lyapunov characteristic exponent $\bar{L}$ and 
the inverse of the average length of the laminar episodes $\langle l\rangle^{-1}$, behave as a power law :

$$
\bar{L} \approx\langle l\rangle^{-1} \sim \mu^{\ln 2 / \ln \lambda_{1}}=\mu^{(z-1) / 2} .
$$

In order to model the presence of a periodic forcing let us extend (1) to the following 2-D mappings :

$$
\begin{aligned}
& x_{i+1}=x_{i}+a\left|x_{i}\right|^{z}+\mu+\varepsilon F\left(x_{i}, \theta_{i}\right) \\
& \theta_{i+1}=\theta_{i}+\Omega+\varepsilon G\left(x_{i}, \theta_{i}\right)
\end{aligned}
$$

where $0 \leqslant \theta \leqslant 2 \pi$ and $-1 \leqslant x<1$ in order to ensure the reinjection process ; $\varepsilon$ characterizes the strength of the coupling; $F(x, \theta)$ and $G(x, \theta)$ are $2 \pi$-periodic functions in $\theta$. Such mappings have been already numerically investigated for particular choices of the functions $F(x, \theta)$ and $G(x, \theta)$ [26]. In the limit of sufficiently small values of the parameters $\mu$ and $\varepsilon$, the dominant route to chaos involves a pair annihilation of tori resulting in the onset of intermittency [27]. This raises the serious problem of bifurcation of invariant tori of differential equations [28, 29] (i.e. invariant circles of mappings) which is much more difficult to handle than the bifurcation theory of periodic orbit. A mathematical study of the saddle-node bifurcation for invariant circles of mappings is reported in [29]. Very restrictive hypotheses have to be satisfied for such a bifurcation to occur. In the case of violation of these hypotheses there is no longer a bifurcation point but a parameter range of fuzziness where chaotic behaviour may even exist. An interesting quantity to compute is thus the shift of the intermittency threshold due to the periodic forcing. For the most common case $z=2$, a perturbative estimate of this shift has been performed in [26]; the critical value $\mu_{\mathrm{c}}$ was found to behave like $\mu_{\mathrm{c}} \sim \alpha(\Omega) \varepsilon^{2}$ (within certain bounds $0 \ll \Omega \ll 2 \pi$ ) in excellent agreement with the results of [30] obtained with a differential equation submitted to a forcing by a two-valued $(\mp \varepsilon, \varepsilon \ll 1)$ step function with period $T$. In the remaining, we will show that such a scaling law comes out quite naturally when using renormalization-group techniques which allow a complete treatment of any universality classes.

When adding a phase as in (8) for $\varepsilon=0$, we generate a " circle " of universal maps $g^{*}(x) \mathrm{e}^{i \theta}$, each characterized by $\theta$. These maps are fixed points of the renormalization operator which shifts the phase of the quantity $\Omega$. For small values of $\varepsilon$ let us look for a perturbation of the form $\varepsilon f(x, \theta) \mathrm{e}^{i(\theta+\varepsilon g(x, \theta))}$ where $f(x, \theta)$ (resp. $\left.g(x, \theta)\right)$ is a $2 \pi$ periodic in $\theta$, and contains as many harmonics as $F(x, \theta)$ (resp. $G(x, \theta))$ does. If $F(x, \theta)$ is monochromatic like in the numerical experiments we will describe in figure 1 to 3 , then $f(x, \theta)$ can be taken in the general form :

$$
f(x, \theta)=\rho(x) \cdot \sin [\theta+\Phi(x)] .
$$

Hence the renormalization operation corresponds to identifying

$$
\alpha^{-1}\left\{g^{*}(\alpha x)+\varepsilon \beta_{\Omega} \rho(\alpha x) \sin \left[\theta+\Omega+\beta_{\Omega}^{\prime}+\Phi(\alpha x)+\mathcal{O}(\varepsilon)\right]\right\} \mathrm{e}^{i(\theta+\Omega+\mathcal{O}(\varepsilon))}
$$

with

$$
\begin{aligned}
\left\{g ^ { * } \left(g^{*}(x)\right.\right. & \left.+\varepsilon f(x, \theta))+\varepsilon f\left(g^{*}(x)+\varepsilon f(x, \theta), \theta+\Omega\right)+\mathcal{O}\left(\varepsilon^{2}\right)\right\} \mathrm{e}^{i(\theta+\Omega+\mathcal{O}(\varepsilon))}= \\
& =\left\{g^{*}\left(g^{*}(x)\right)+\varepsilon\left[g^{* \prime}\left(g^{*}(x)\right) \cdot f(x, \theta)+f\left(g^{*}(x), \theta+\Omega\right)\right]+\mathcal{O}\left(\varepsilon^{2}\right)\right\} \mathrm{e}^{i(\theta+\Omega+\mathcal{O}(\varepsilon))} .
\end{aligned}
$$

When $\varepsilon$ is small, on using (2) and (9), we obtain from (10) and (11) a system of two coupled 
equations for $\rho(x)$ and $\Phi(x)$ (at the first order in $\varepsilon$ )

$$
\begin{aligned}
\alpha^{-1} \beta_{\Omega} \rho(\alpha x) \cos \left[\Omega+\beta_{\Omega}^{\prime}+\right. & \Phi(\alpha x)]= \\
& =\rho(x) g^{*^{\prime}}\left(g^{*}(x)\right) \cos (\Phi(x))+\rho\left(g^{*}(x)\right) \cos \left[\Omega+\Phi\left(g^{*}(x)\right)\right] \\
\alpha^{-1} \beta_{\Omega} \rho(\alpha x) \sin \left[\Omega+\beta_{\Omega}^{\prime}+\right. & \Phi(\alpha x)]= \\
& =\rho(x) g^{*^{\prime}}\left(g^{*}(x)\right) \sin (\Phi(x))+\rho\left(g^{*}(x)\right) \sin \left[\Omega+\Phi\left(g^{*}(x)\right)\right] .
\end{aligned}
$$

Carrying out a calculation of (12) on using series-expansion techniques (as well as polynomial interpolations for $\rho(x)$ and $\Phi(x))$ and the expression (3) for $g^{*}(x)$, we find

$$
\beta_{\Omega}=2^{z /(z-1)} \cos (\Omega / 2), \quad \beta_{\Omega}^{\prime}=-\Omega / 2
$$

where $0<\Omega<\pi$ as a consequence of the global invariance of the problem under a change in the signe of $\Omega$.

At this point, let us remark that any perturbation of the phase by terms of order $\varepsilon(g(x, \theta))$ does not enter our renormalization-group analysis since it contributes to order $\varepsilon^{2}$ (and not $\varepsilon$ ) to the equation for the modulus. This means that the universal properties of the transition from quasiperiodicity to chaos via intermittency should not be affected by the presence of a coupling $G(x, \theta)$ in the equation for the phase in (8). Such a term is responsible for the possible existence of frequency lockings on the original torus since the winding number is no longer kept fixed to an irrational value $\Omega$.

From (10) and (11), it is clear that the perturbation we have considered is not an eigenvector of the linear part $D R$ of the renormalization operator since

$$
\begin{aligned}
D R f(x, \theta) & =\alpha^{-1} \beta_{\Omega} \rho(\alpha x) \cdot \sin \left[\theta+\Omega+\beta_{\Omega}^{\prime}+\Phi(\alpha x)\right] \\
& =\alpha^{-1} 2^{z /(z-1)-1} \rho(\alpha x)\{\sin [\theta+\Phi(\alpha x)]+\sin [\theta+\Omega+\Phi(\alpha x)]\} .
\end{aligned}
$$

Nevertheless, from ergodicity, one can define an " average » expansion factor by noticing [17] that the second term on the right-hand side of (14) does not contribute when one averages over successive iterations of $D R$ [31]. Thus one gets an « average » relevant eigenvalue.

$$
\lambda_{2}=2^{1 /(z-1)}=\alpha
$$

which lies out of the unit circle and is associated with a new (average) unstable direction for the fixed point $g^{*}(x)$ as introduced by the periodic forcing. Then the order parameter depends on $\mu$ and the amplitude of the external field $\varepsilon$, according to the scaling form [17]

$$
\langle l\rangle^{-1}=\varepsilon^{\chi} \cdot \Lambda\left(\mu / \varepsilon^{\gamma}\right)
$$

with $\Lambda(y)$ a universal function, and $\chi$ and $\gamma$ universal exponents which can be easily computed from the eigenvalues $\lambda_{1}$ and $\lambda_{2}$ as given by (6) and (15) respectively :

$$
\begin{aligned}
& \chi=\ln 2 / \ln \lambda_{2}=z-1 \\
& \gamma=\ln \lambda_{1} / \ln \lambda_{2}=z
\end{aligned}
$$

From (7) $\Lambda(y)$ fits the asymptotic behaviour $\Lambda(y) \sim y^{x / \gamma}$ for large $y$.

These strikingly simple expressions of the universal exponents $\gamma, \chi$ with respect to $z$ deserve an experimental test. In figures 1 and 2 we first report on numerical investigations of the 2-D mapping (8) in the most common case $z=2$. Exploring several irrational values of $\Omega$ and different couplings $F(x, \theta)$ and $G(x, \theta)$, we have computed $\langle l\rangle^{-1}$ for various values of $\varepsilon$. As shown in figure la with 

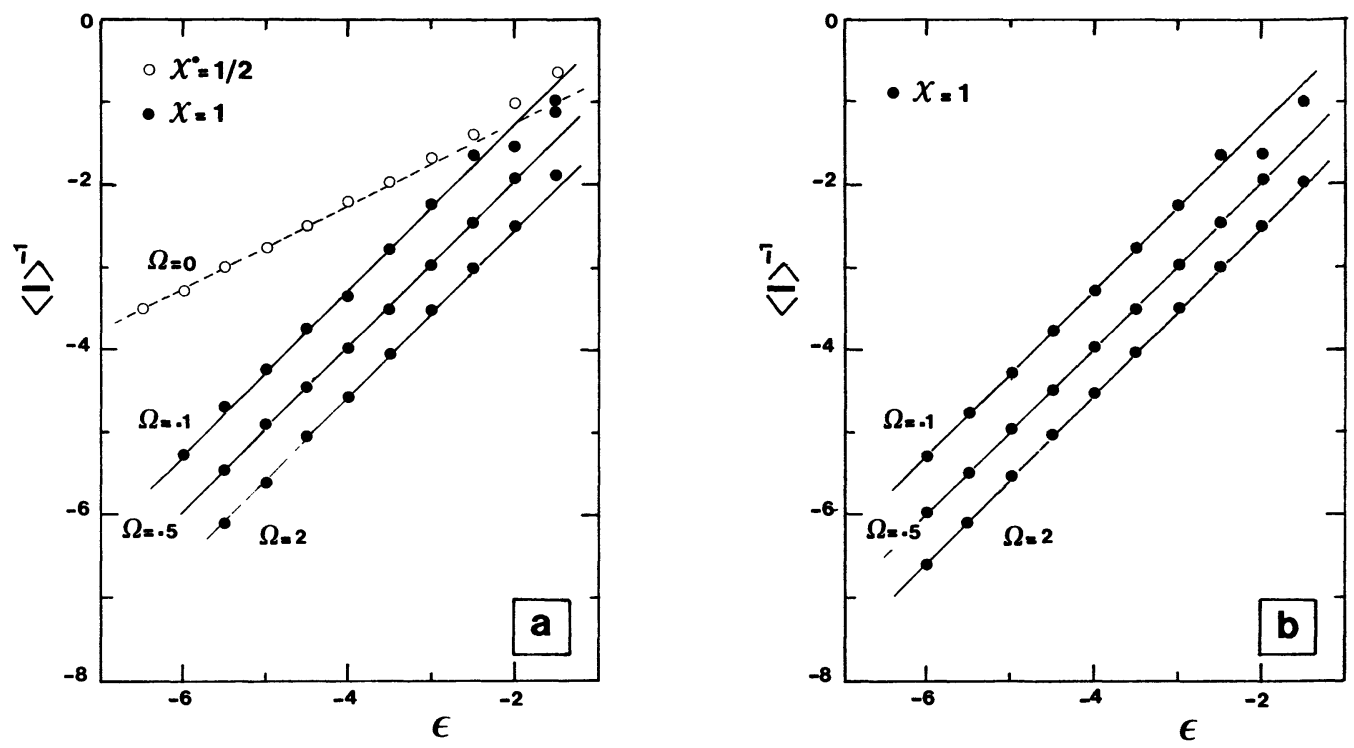

Fig. 1. - A $\log _{10}-\log _{10}$ plot of $\langle l\rangle^{-1}$ versus $\varepsilon$ as computed with the 2-D mappings (8) for an acceptance gate $|x|<10^{-1}$ and different values of $\Omega ; z=2.0, a=1.75, \mu=0.0 ; F(x, \theta)=\cos \theta$ and (a) $G(x, \theta)=$ $0.0,(\mathrm{~b}) G(x, \theta)=x$. The continuous lines correspond to the prediction given by (17).

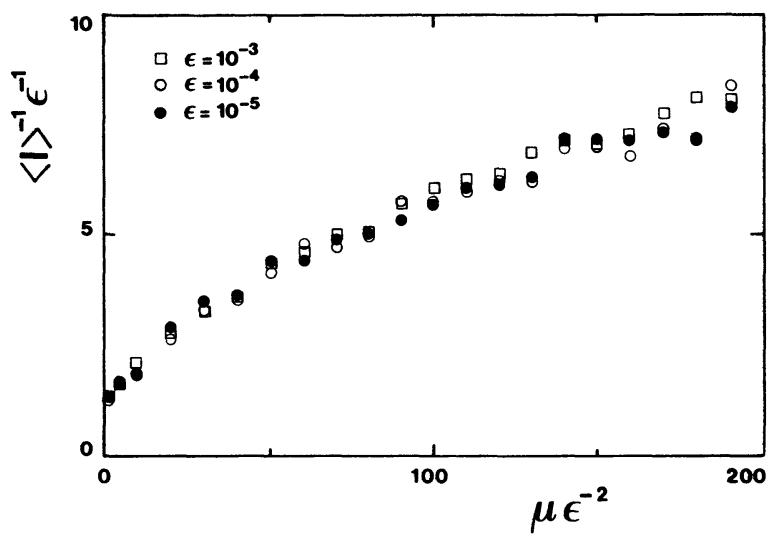

Fig. 2. - Numerical determination of the scaling function $\Lambda(y)$ defined in (16). The quantity $\langle l\rangle^{-1} \varepsilon^{-\chi}$ is plotted against $y=\mu / \varepsilon^{\gamma}$ at each of three values $\varepsilon=10^{-3}, 10^{-4}, 10^{-5} \cdot\langle l\rangle^{-1}$ is calculated through the iterations of $(8)$ with the same model parameters as in figure la $(\Omega=0.5)$.

$F(x, \theta)=\cos \theta$ and $G(x, \theta)=0$, the data are well fitted with $\chi=1$ as given by (17) (for $\Omega=0$, we recover $\chi^{*}=1 / 2$ as obtained without periodic forcing). The same good agreement exists when introducing the coupling $G(x, \theta)=x$ as illustrated in figure $1 \mathrm{~b}$ which confirms our prediction that the critical exponents are not affected by the presence of a coupling in the equation for the phase. 
To check the existence of the scaling function $\Lambda(y)$ of (16), we use the values of $\chi$ and $\gamma$ obtained from (17) and (18) to plot $\langle l\rangle^{-1} \varepsilon^{-\chi}$ as a function of $\mu \varepsilon^{-\gamma}$. The results are shown in figure 2 for three different values of the strength of the coupling : $\varepsilon=10^{-3}, 10^{-4}, 10^{-5}$. The data for those three different values of $\varepsilon$ all fall on a universal curve in the vicinity of the chaotic threshold. Let us emphasize that although this curve depends on $\Omega$, it is always zero for some $y_{0}<0$. This allows us to conclude that the shift of the intermittency threshold due to the periodic forcing scales like $\mu_{\mathrm{c}} \approx \varepsilon^{\gamma}=\varepsilon^{2}$, which confirms the analysis in [26,30]. More generally such a shift is predicted to scale like :

$$
\mu_{\mathrm{c}} \approx \varepsilon^{z} .
$$

In figure 3, we present the results of numerical simulations of (8) for different values of $z$, namely $z=2,3,4$. A remarkable agreement with the predictions (17) and (18) is found for the whole set of universality classes, which attests of the generality of our theoretical analysis.

To conclude, let us notice that from a comparison of $\lambda_{2}$ as given by (15) with the " average " relevant eigenvalue $\lambda_{\text {noise }}$ obtained in the presence of a random noise [12-14] :

$$
\lambda_{\text {noise }}=2^{(z+1) / 2(z-1)}
$$

it results that $\lambda_{2}<\lambda_{\text {noise }}$ for $z>1$, which infers that intermittency is much less unstable to the presence of a periodic forcing than to random fluctuations.

\section{Acknowledgments.}

We are very grateful to $\mathbf{P}$. De Kepper for a careful reading of the manuscript.

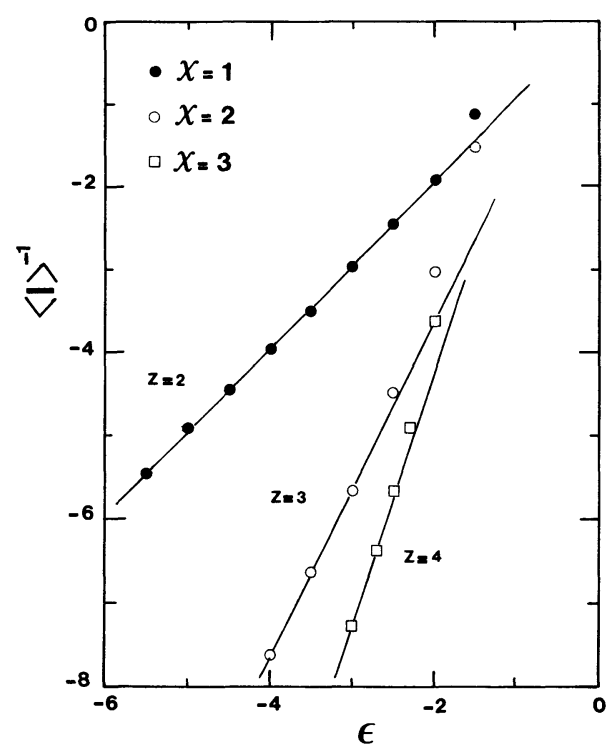

Fig. 3. - A $\log _{10}-\log _{10}$ plot of $\langle l\rangle^{-1}$ versus $\varepsilon$ as computed with the 2-D mappings (8) for an acceptance gate $|x|<10^{-1}$ and different values of $z ; F(x, \theta)=\cos \theta$ and $G(x, \theta)=0.0 ; \mu=0.0, \Omega=0.5$. Solid dots : $z=2, a=1.75$; open circles : $z=3, a=1.5$; squares : $z=4, a=1.3$. The continuous lines correspond to the predictions given by (17). 


\section{References}

[1] Abraham, N. B., Gollub, J. P. and Swinney, H. L., Meeting report, Physica D 11 (1984) 252 and references therein.

[2] Eckmann, J. P., Rev. Mod. Phys. 53 (1981) 643 and references therein.

[3] OTt, E., Rev. Mod. Phys. 53 (1981) 655 and references therein.

[4] MA, S. K., Modern Theory of Critical Phenomena (Benjamin, Reading, Mass.) 1976.

[5] Wilson, K. G., Rev. Mod. Phys. 55 (1983) 583.

[6] Feigenbaum, M. J., J. Stat. Phys. 19 (1978) and 21 (1979) 669.

[7] Coullet, P. and Tresser, C., J. Physique Colloq. 39 (1978) C5-25.

[8] Tresser, C. and Coullet, P., C. R. Acad. Sci., Paris 287 (1978) 577.

[9] Collet, P. and Eckmann, J. P., Iterated Maps on an Interval as Dynamical Systems (Birkhauser, Boston) 1980.

[10] Manneville, P. and Pomeau, Y., Phys. Lett. A 75 (1979) 1 and Commun. Math. Phys. 74 (1980) 189.

[11] Eckmann, J. P., Thomas, L. and Wittwer, P., J. Phys. A 14 (1981) 3153.

[12] Hirsch, J. E., Huberman, B. A. and Scalapino, D. J., Phys. Rev. A 25 (1982) 519.

[13] Hirsch, J. E., Nauenberg, M. and Scalapino, D. J., Phys. Lett. A 87 (1982) 391.

[14] Hu, B. and RudNick, J., Phys. Rev. Lett. 48 (1982) 1645.

[15] Coullet, P., in " Chaos and Statistical Methods ", edited by E. Kuramoto, Springer Series in Synergetics Vol. 24 (Springer-Verlag, Berlin) 1984.

[16] ArnÉODO, A., in Fluctuations and Sensitivity in Nonequilibrium Systems, edited by W. Horsthemke and D. K. Kordepudi (Springer-Verlag, Berlin) 1984, p. 187.

[17] Argoul, F. and ArnÉodo, A., "Lyapunov exponents and phase transitions in dynamical systems " - preprint 1985 - To appear in Lecture Notes in Mathematics.

[18] Crutchfield, P. and Huberman, B. A., Phys. Lett. A 77 (1980) 407.

[19] Crutchfield, J. P., Nauenberg, M. and Rudnick, J., Phys. Rev. Lett. 46 (1981) 933.

[20] Schraiman, B., Wayne, C. E. and Martin, P. C., Phys. Rev. Lett. 46 (1981) 935.

[21] Crutchfield, J. P., Farmer, J. D. and Huberman, B. A., Phys. Rep. 92 (1982) 47 and references therein.

[22] Arnéodo, A., Coullet, P. H. and Spiegel, E. A., Phys. Lett. A 94 (1983) 1.

[23] ARnÉodo, A., Phys. Rev. Lett. 53 (1984) 1240 and 54 (1985) 86.

[24] ARgoul, F. and ARnÉodo, A., " From quasiperiodicity to chaos : an unstable scenario via perioddoubling bifurcations of tori ", preprint 1984, to appear in J. Mec. Theor. Appl.

[25] Kaneko, K., Prog. Theor. Phys. 69 (1983) 1806.

[26] DaIDo, H., Prog. Theor. Phys. 71 (1984) 402.

[27] For experimental evidences of intermittency from torus we refer the reader to : GolLuB, J. P. and Benson, S. V., J. Fluid. Mech 100 (1980) 449.

MAurer, J. and LibChaber, A., J. Physique Lett. 41 (1980) L-515.

Sano, M. and SaWADa, Y., preprint.

[28] Chenciner, A. and Iooss, G., Arch. Ration. Mech. Anal. 69 (1979) 109 and 71 (1979) 301.

[29] Chenciner, A., in Chaotic Behavior in Deterministic Dynamical Systems, edited by G. Iooss, R. G. H. Helleman and R. Stora, Les Houches Summer School Proceedings 36 (North-Holland, Amsterdam) 1983; " Hamiltonian-like phenomena in saddle-node bifurcations of invariant curve for plane diffeomorphisms ", Preprint 1984 and references therein.

[30] Caroli, B., Caroli, C. and Roulet, B., Phys. Lett. A 94 (1983) 117.

[31] On the contrary to the period-doubling case [23, 24], one has to take an arithmetical instead of a geometric mean. 